\title{
LXXXI. Rotation of elastic bodies and the principle of relativity
}

\section{P.J. Daniell}

To cite this article: P.J. Daniell (1915) LXXXI. Rotation of elastic bodies and the principle of relativity, Philosophical Magazine Series 6, 30:180, 754-761, DOI: $\underline{10.1080 / 14786441208635455}$

To link to this article: http://dx.doi.org/10.1080/14786441208635455

曲 Published online: 08 Apr 2009.

Submit your article to this journal ๘

Џ Article views: 3

Q View related articles $\sqsubset$ 
LXXXI. Rotation of Elastic Bodies and the Principle of Relativity. By P. J. Daniell, Assistant-Professor of Mathematics, Rice Institute, Houston, Tex. ${ }^{*}$

T $\mathrm{T}$ is well known that the rotation of a "rigid" body 1 about a stationary axis is inconsistent with the Principle of Relativity. In fact, the circle aloing which any part moves is contracted while the radius is unaltered. It has been suggested that a rotating circular disk might buckle, but it could then no longer be regarded as rigid. Herglotz, in a paper on the mechanics of deformable bodies $t$, has shown a method by which problems relating to elastic bodies may be solved. The method depends on the variation of the integral of a potential function of the rest-deformations; that is to say, if each elementary part of the body is referred to axes with respect to which it is at rest, the deformations in terms of these new axes are the rest-deformations. Here this method has been used to consider the question of the rotation of an isotropic elastic body. It is found that even when the elastic constants are infinite, rotation is possible, although the rest-deformations are not zero. The body is not "rigid" in the absolute sense of Born's definition. It might perhaps be called semi-rigid. By some the Principle of Relativity has been extended so as to include the assumption that even elastic waves cannot be propagated with a volocity greater than that of light; then the ratios of the elastic constants to the density cannot exceed the square of the velocity of light. The elastic ratios of all material bodies, even of su-called "incompressible" fluids, do not approach this value. But in electron theory the "semirigid" body whose rotation is not inconsistent with the Principle of Relativity may be even more useful a conception than Born's rigid electron.

Only small deformations will be considered, and the square of the ratio of the velocity of any part to the velocity of light will be taken as small. Squares and products of such quantities will be neglected throughout.

Let $r$ denote a position vector in the undeformed body referred to a system $S$;

$r+b$ denotes the corresponding deformed position vector referred to space-axes rotating with the body;

$r^{\prime}$ denotes the corresponding deformed position vector referred to $\mathrm{S}$.

* Communicated by the Author.

+ Herglotz, "Mechanil des Deformierbaren Körpers," Annalen der Physik, xxxvi. p. 500. 


$$
\text { Rotation of Elastic Bodies. }
$$

If $u^{\prime}$ is the vector velocity of an element,

Let

$$
d r^{\prime}=d r+d b+u^{\prime} d t
$$

$$
d r_{0}=d r+d a
$$

denote the differential of the corresponding position vector obtained by making a Lorentz transformation to space-time axes with regard to which the element is at rest.

Then $d a$ is the rest-deformation differential vector.

The Lorentz transformation gives

where

$$
d r_{0}=d r^{\prime}+\frac{\alpha u^{\prime}}{c^{2}}\left(u^{\prime} \cdot d r^{\prime}\right)-\beta u^{\prime} d t
$$

$$
\beta=\frac{1}{\sqrt{1-\frac{u^{12}}{c^{2}}}}, \quad \alpha=\frac{\beta^{2}}{\beta+1},
$$

and $c$ is the velocity of light,

$$
\text { or } \quad d r_{0}=d r+d b+\frac{\alpha u^{\prime}}{c^{2}}\left(u^{\prime} \cdot d r+d b\right) \text {. }
$$

Instead of $u^{\prime}$ consider

$$
u=(\omega \times r),
$$

where $\omega$ is the rotation vector and $(\omega \times r)$ is a vector product.

Then, neglecting squares of small quantities,

$$
\begin{aligned}
& d r_{0}=d r+d b+\frac{u}{2 c^{2}}(u \cdot d r), \\
& d a=d b+\frac{u}{2 c^{2}}\left(u \cdot d r^{2}\right) .
\end{aligned}
$$

Again,

$$
\begin{aligned}
d t_{0} & =-\frac{\beta}{c^{2}}\left(u^{\prime} \cdot d r^{\prime}\right)+\beta d t, \\
& =-\frac{\beta}{c^{2}}\left(u^{\prime} \cdot d r+d b\right)+\beta\left(1-\frac{u^{\prime 2}}{c^{2}}\right) d t .
\end{aligned}
$$

If we consider $t_{0}$ as changing while $r$, and therefore $b$, is fixed (i.e., uniform rotation),

$$
\begin{aligned}
d t_{0} & =\beta\left(1-\frac{u^{\prime 2}}{c^{2}}\right) d t \\
& =\left(1-\frac{1}{2} \frac{u^{\prime 2}}{c^{2}}\right) d t, \quad \text { or K } d t \text { let us say. }
\end{aligned}
$$


The potential function depends only on the rest-deformations $a$. It can be seen that

(i.) $\operatorname{div} a=\operatorname{div} b+\frac{u^{2}}{2 c^{2}}$,

(ii.) curl $a=\operatorname{curl} b$,

(iii.) $\operatorname{grad} a_{x}=\operatorname{grad} b_{x}+\left(\frac{u_{x}}{2 c^{2}}\right) \cdot u$,

(iv.) at the surface if $\delta n$ denotes an element of normal to the surface, and if $n_{1}$ is a unit vector in the direction of the normal

$$
\frac{\partial a}{\partial n}=\frac{\partial b}{\partial n}+\left(n_{1} \cdot u\right) \frac{u}{2 c^{2}} .
$$

Only bodies symmetrical about their axes of rotation will be considered, and then $u$ is perpendicular to $n_{1}$.

$$
\begin{aligned}
& \text { Or } \\
& \text { Let us put* } \\
& \frac{\partial \alpha}{\partial n}=\frac{\partial b}{\partial n} \text {. } \\
& e_{x x}=\frac{\partial a_{x}}{\partial x}, \quad e_{y y}=\frac{\partial a_{y}}{\partial y}, \quad e_{z z}=\frac{\partial a_{z}}{\partial z} \\
& e_{y z}=\frac{1}{2}\left(\frac{\partial a_{z}}{\partial y}+\frac{\partial a_{y}}{\partial z}\right), \quad e_{z x}=\frac{1}{2}\left(\frac{\partial a_{x}}{\partial z}+\frac{\partial a_{z}}{\partial x}\right), \quad e_{x y}=\frac{1}{2}\left(\frac{\partial a_{y}}{\partial x}+\frac{\partial a_{x}}{\partial y}\right) .
\end{aligned}
$$

The ordinary potential function is given by $W^{\prime}$ where

$$
2 W^{\prime}=(\lambda+2 \mu)\left(e_{x x}+e_{y y}+e_{z z}\right)^{2}+4 \mu\left(e_{y z}^{2}+e_{z x}^{2}+e_{x y}^{2}-e_{y y} e_{z z}-e_{z z} e_{x x}-e_{x x} e_{y y}\right) \text {. }
$$

This can be reduced to the form

$$
2 W^{\prime}=\lambda(\operatorname{div} a)^{2}-\mu(\operatorname{curl} a)^{2}+2 \mu\left[\left(\operatorname{grad} a_{x}\right)^{2}+\left(\operatorname{grad} a_{y}\right)^{2}+\left(\operatorname{grad} a_{z}\right)^{2}\right] \text {. }
$$

The "action" would be given by 'T-W', where $\mathrm{T}$ is the kinetic energy,

$$
\mathrm{T}=\frac{1}{2} \rho u^{\prime 2}=\frac{1}{2} \mathrm{M} \frac{u^{\prime 2}}{c^{2}}, \quad \text { if } \mathrm{M}=\rho c^{2} .
$$

We can bring this kinetic energy into the expression of the potential function by assuming it to be

$$
\mathrm{W}=-\mathrm{M}-\mathrm{W}^{\prime}
$$

per unit 4-volume in $\left(x_{0} y_{0} z_{0} t_{0}\right)$ space.

$\mathrm{W}=-\frac{1}{2} \lambda(\operatorname{div} a)^{2}+\frac{1}{2} \mu(\operatorname{curl} a)^{2}-\mu\left[\left(\operatorname{grad} a_{x}\right)^{2}+\left(\operatorname{grad} a_{y}\right)^{2}+\left(\operatorname{grad} a_{z}\right)^{2}\right]-\mathrm{M}$.

$\lambda, \mu$ are the ordinary elastic constants, $\mathrm{M}=c^{2} \times$ the density $\rho$.

$$
\text { * Love, 'Elasticity,' 2nd edition. }
$$


The required differential equations are found by making the variation

$$
\begin{array}{ll}
\delta \iiint \int \mathrm{W} d s d y d z d t_{0}=0, \\
\text { or } & \delta \iiint \int \mathrm{W} d x d y d z(\mathrm{~K} d t)=0, \\
\text { or } & \delta \iiint \mathrm{WK} d x d y d z=0
\end{array}
$$

if the rotation is uniform.

In the elastic terms of $W, K$ can be put equal to 1 ; $K$ is retained only when it is multiplied by $M$, and then the kinetic energy term appears.

This variation is 0 for all possible variations $\delta b$ if

$\lambda \operatorname{grad} \operatorname{div} a+\mu$ curl curl $a+2 \mu(\operatorname{div} \operatorname{grad}) a+\mathrm{M} \operatorname{grad} \frac{u^{2}}{2 c^{2}}=0$ throughout the volume, and if at the surface

$$
\lambda n_{1} \operatorname{div} a+\mu\left(n_{1} \times \operatorname{curl} \alpha\right)+2 \mu \frac{\partial \alpha}{\partial n}=0 .
$$

These are exactly the equations which occur in the Newtonian mechanics (cf. Love, 'Elasticity'); but they refer to $a$, the rest-deformation, not $b$, the actual deformation.

We must use the transformations (i. to iv.) above. Then $\lambda \operatorname{grad} \operatorname{div} b+\mu \operatorname{curl} \operatorname{curl} b+2 \mu(\operatorname{div} \operatorname{grad}) b$

$$
\begin{aligned}
+(\lambda+\mathrm{M}) \operatorname{grad} \frac{u^{2}}{2 c^{2}}+2 \mu(\operatorname{grad} \cdot u) \frac{u}{2 c^{2}}=0, & \\
(\operatorname{grad} \cdot u) u & =(u \cdot \operatorname{grad}) u+u \operatorname{div} u, \\
\operatorname{div} u & =\operatorname{div}(\omega \times r)=0, \\
(u \cdot \operatorname{grad}) u & =(\omega \times u), \\
\quad \frac{1}{2} \operatorname{grad} u^{2} & =(u \cdot \operatorname{grad}) u+(u \times \operatorname{curl} u) .
\end{aligned}
$$

But curl $u=2 \omega$

$$
\begin{aligned}
& \text { or }(u \times \operatorname{curl} u)=-2(\omega \times u)=-2(u \cdot \operatorname{grad}) u, \\
& \quad \text { so }(\operatorname{grad} . u) u=-\frac{1}{2} \operatorname{grad} u^{2} .
\end{aligned}
$$

Hence the volume equation becomes

(v.) $(\lambda+2 \mu) \operatorname{grad} \operatorname{div} b-\mu \operatorname{curl}$ curl $b$

and the surface equation becomes

$$
+(\lambda-\mu+M) \operatorname{grad} \frac{u^{2}}{2 c^{2}}=0,
$$

(vi.) $\lambda n_{1} \operatorname{div} b+\mu\left(n_{1} \times \operatorname{curl} b\right)$

$$
+2 \mu \frac{\partial \zeta}{\partial n}+\lambda n \frac{u^{2}}{2 c^{2}}=0 .
$$


These are the same as the ordinary equations obtained for a body with the same elastic constants $\lambda, \mu$, with density $\frac{\lambda-\mu+M}{c^{2}}$ instead of $\rho$ or $\frac{M}{c^{2}}$, and acted on by a surface force normal to the surface $=\lambda \frac{u^{2}}{2 c^{2}}$ pressing in wards. The solutions in any particular problems can be found by the ordinary methods, if these two changes are made.

For the general case Betti's Reciprocation Theory * can be used. We shall denote by $\bar{e}_{x x}$ the average value, not of $e_{x x}$ as defined above in terms of $a$, but of an $e_{x x}=\frac{\partial b_{x}}{\partial x}$ defined in terms of the actual deformation. There will be apparent volume forces $(\mathrm{M}+\lambda-\mu) \operatorname{grad} \frac{u^{2}}{2 \varepsilon^{2}}$ and apparent normal surface forces $\lambda \frac{u^{2}}{2 e^{2}}$ inwards.

Let E denote Young's modulus, $\sigma$ Poisson's ratio, $V$ the volume.

$$
\begin{gathered}
\vec{e}_{x x}=\frac{\mathrm{M}+\lambda-\mu}{\mathrm{EV}} \iiint_{0}^{0}\left[x \frac{d}{d x}\left(\frac{u^{2}}{2 c^{2}}\right)-\sigma y \frac{d}{d y}\left(\frac{u^{2}}{2 c^{2}}\right)\right. \\
\left.-\sigma z \frac{d}{d z}\left(\frac{u^{2}}{2 c^{2}}\right)\right] d x d y d z \\
-\frac{\lambda}{\mathrm{EV}} \iint \frac{\mathrm{V}^{2}}{2 c^{2}}(x l-\sigma y m-\sigma z n) d \mathrm{~S},
\end{gathered}
$$

where $d \mathbf{S}$ denotes an element of surface,

$l, m, n$ are the direction cosines of the outward drawn normal.

But $\iiint x \frac{d}{d x}\left(\frac{u^{2}}{2 c^{2}}\right) d x d y d z=\iint \frac{u^{2}}{2 c^{2}} x l d s-\iint \frac{u^{2}}{2 c^{2}} d x d y d z$

Then

$$
\begin{gathered}
\vec{e}_{x x}=\frac{\mathrm{M}-\mu}{\mathrm{EV}} \iint\left[x \frac{d}{d x}\left(\frac{u^{2}}{2 c^{2}}\right)-\sigma y \frac{d}{d y}\left(\frac{u^{2}}{2 c^{2}}\right)\right. \\
\left.-\sigma z \frac{d}{d z}\left(\frac{u^{2}}{2 c^{2}}\right)\right] d x d y d z \\
-\frac{\lambda}{\mathrm{EV}} \iiint \frac{u^{2}}{2 c^{2}}(1-2 \sigma) d z d y d z .
\end{gathered}
$$

But $\frac{d}{d x}\left(\frac{u^{2}}{2 c^{2}}\right)=\frac{1}{c^{2}}\left[\left(\omega_{y}^{2}+\omega_{z}^{2}\right) x-\omega_{z} \omega_{x} z-\omega_{y} \omega_{x} y\right]$.

$$
\text { * Love, 'Elasticity' (2nd ed.), p. } 170 .
$$


Elastic Bodies and the Principle of Relativity.

Let $x=0, y=0, z=0$ be principal planes of the body

and let

$$
k_{x}^{2}=\mathrm{V} \iint_{x} x^{2} d x d y d z \text {, etc. }
$$

Further, let $\mathrm{O} z$ be the axis of rotation and suppose the body to be symmetrical about this axis.

Then

$$
\begin{aligned}
& \omega_{x}=0, \omega_{y}=0, \omega_{z}=\omega, \\
& k_{x}{ }^{2}=k_{y}{ }^{2}=\frac{1}{2} k_{1}{ }^{2} .
\end{aligned}
$$

Then

$$
\begin{aligned}
\tilde{e}_{x x} & =\bar{e}_{y y}=\frac{\omega^{2} k_{1}^{2}}{2 c^{2} \mathrm{E}}[(\mathrm{M}-\mu)(1-\sigma)-\lambda(1-2 \sigma)] \\
& =\frac{\omega^{2} k_{1}^{2}}{2}\left[\frac{M(1-\sigma)}{c^{2} \mathrm{E}_{1}}-\frac{1}{2 c^{2}}\right] .
\end{aligned}
$$

Newtonian mechanics would give the first term only, and the average $\bar{e}_{x x}, \bar{e}_{y y}$ are less according to the Principle of Relativity.

$$
\begin{aligned}
\bar{e}_{z z} & =\frac{\omega^{2}{k_{1}}^{2}}{2 c^{2} \overline{\mathrm{E}}}[-2 \sigma(\mathrm{M}-\mu)-\lambda(1-2 \sigma)] \\
& =-\omega^{2}{k_{1}}^{2} \frac{\mathrm{M} \sigma}{c^{2} \overline{\mathrm{E}}} .
\end{aligned}
$$

This is the same as in Newtonian mechanics.

Similarly, using Betti's theorem it can be shown that

$$
\bar{e}_{y z}=\bar{e}_{z x}=\bar{e}_{x y}=0 \text {, as in Newtonian mechanics. }
$$

We can define a "semi-rigid" body as one in which $\mathrm{E}$ is infinite, not only as compared with $\rho$, but with $\rho c^{2}$ or M.

Then

$$
\begin{aligned}
& \bar{e}_{z z}=0, \bar{e}_{y z}=\bar{e}_{z x}=\bar{e}_{x y}=0, \\
& \bar{e}_{x x}=\bar{e}_{y y}=-\frac{\omega^{2} k_{1}^{2}}{4 c^{2}} .
\end{aligned}
$$

Thus a body with infinite elastic constants, or infinitesimal density, can rotate, but it does not remain "rigid" in the absolute sense.

$$
\begin{aligned}
\overline{\operatorname{div} a} & =\overline{\operatorname{div} b}+\frac{\omega^{2} r^{2}}{2 c^{2}} \\
& =\bar{e}_{x x}+\bar{e}_{y y}+\bar{e}_{z z}+\frac{\omega^{2} / k_{1}{ }^{2}}{2 c^{\ddot{4}}} \\
& =\omega^{2} k_{1}{ }^{2}\left[\frac{\mathrm{M}(1-\sigma)}{c^{2} \mathrm{E}}-\frac{\mathrm{M} \sigma}{c^{2} \mathrm{E}}\right] \\
& =\omega^{2} k_{1}{ }^{2} \frac{\mathrm{M}(1-2 \sigma)}{c^{2} \mathrm{E}} .
\end{aligned}
$$



This is 0 when $\frac{E}{M}$ is infinite, but $a$ itself is not necessarily
0 throughout.

For all known material bodies, although $\mathrm{E} / \rho$ is large, $\mathrm{E} / \mathrm{M}=\rho e^{2}$ is very small. Hence no experiments on the rotation of bodies could decide between Newtonian and Relative mechanics.

Two interesting special cases can be worked out by methods practically the same as in Newtonian mechanics (cf. Love, 'Elasticity' (2nd ed.), p. 144).

Case 1.--Long cylinder, assuming a uniform longitudinal oxtension $e$ so that $\omega=e z$.

When $r=a$ the relative radial extension $\frac{n}{a}$ is given by

$$
\begin{aligned}
& \frac{u}{a}=\frac{\omega^{2} a^{2}}{4 c^{2}}\left[\frac{\mathrm{M}(1-\sigma)}{\mathrm{E}}-\frac{1}{2}\right] \\
& e=-\frac{\omega^{2} a^{2}}{2 c^{2}} \frac{\mathrm{M} \sigma}{\mathrm{E}} .
\end{aligned}
$$

For a "semirigid" body

$$
e=0, \quad \frac{u}{a}=-\frac{\omega^{2} a^{2}}{8 c^{2}} .
$$

Case 2.-Thin disk, with the same assumptions as in Love's ' Elasticity.'

When $r=a, z=0$,

$$
\frac{u}{a}=\frac{\omega^{2} a^{2}}{4 c^{2}}\left[\frac{\mathrm{M}(1-\sigma)}{\mathrm{E}}-\frac{1}{2}+\frac{\mathrm{M} \sigma(1+\sigma)}{\mathrm{E}} \frac{2 l^{2}}{3 a^{2}}-\sigma \frac{l^{2}}{a^{2}}\right] \text {. }
$$

When $r=0, z=l$,

$$
\frac{w}{l}=-\frac{\omega^{2} a^{2}}{8 c^{2}}\left[\frac{\lambda(7 \lambda+6 \mu)}{(\lambda+\mu)(3 \lambda+2 \mu)} \cdot \frac{\mathrm{M}}{2 \mu}-3 \sigma\right] \text {. }
$$

For a " semirigid" body

$$
\begin{aligned}
\frac{u}{a} & =-\frac{\omega^{2} a^{2}}{\delta c^{2}}\left(1+2 \sigma \frac{l^{2}}{a^{2}}\right), \\
\frac{w}{l} & =\frac{\omega^{2} a^{2}}{8 c^{2}} 3 \sigma .
\end{aligned}
$$

According to some an electron is supposed to possess no non-electromagnetic mass. In this case $M$ would be infinitesimal and the electron would appear, at first sight, to be "semirigid" rather than absolutely "rigid." Such an 
electron or even a positive ion (of smaller radius so as to possess greater mass) could rotate and something like a magneton would be the result, even if the elastic constants were not supposed to be infinite. Actually, however, the electromagnetic "potential" energy will produce effects analogous to those due to a mass density varying from the centre to the circumference. By supposing $\lambda$ and $\mu$ to be infinite, the "semirigid " rotating electron (electronmagneton) could still be used as an hypothesis consistent with the Principle of Relativity.

LXXXII. On the Solution of Certain Problems of TwoDimensional Physics. By J. R. WILToN, M.A., D.Sc., Assistant Lecturer in Mathematies at the University of Sheffield*.

1. GENERAL method of solution of certain types of A physical problem, in which the boundary considered consists of a single analytical curve, may be founded on the obvious remark $\uparrow$ that the transformation

$$
x+\iota y=\mathrm{X}(\tau)+\iota \mathrm{Y}(\tau),
$$

in which $\tau=\eta-\iota \xi$, and $\mathrm{X}$ and $\mathrm{Y}$ are real when $\tau$ is real, makes the real axis in the $\tau$ plane correspond to the curve

$$
x=\mathrm{X}(\eta), y=\mathrm{Y}(\eta), . \quad . \quad . \quad .
$$

in the $x+\iota y$ plane. We may therefore take the equation of any analytical boundary in the form

or, if $\theta=\eta+\iota \xi$, we have

$$
\xi=0, . \text {. . }
$$

$$
\theta=\tau \text {. }
$$

For the sake of brevity, we shall denote $\mathrm{X}(\eta)$ by $\mathrm{X}, \mathrm{X}(\boldsymbol{\tau})$ by $X_{1}$, and $X(\theta)$ by $X_{2}$, with a similar notation in the case of $\mathrm{Y}$.

In the simplest type of problem we are required to determine a function $\psi$ from

$$
\nabla^{2} \psi=0
$$

together with the conditions $\psi=f(\eta), \frac{\partial \psi}{\partial n}=F(\eta)$ on the boundary, where $d n$ is an element of the outward drawn normal. The solution is

$$
\psi=\frac{1}{2}\{f(\theta)+f(\tau)\}+\frac{1}{2 \iota} \int_{\tau}^{\theta}\left(\mathrm{X}^{\prime 2}+\mathrm{Y}^{\prime 2}\right)^{\frac{1}{2}} \mathrm{~F}(\eta) d \eta .
$$

* Communicated by the Author.

+ Cf. Forsyth, 'Theory of Functions,' $§ 265$, p. 624 ('nd edition); also Jeans, ' Electricity,' p. 264.

Phil. Mag. S.6. Vol. 30. No. 180. Dec. 1915. 3 D 